\title{
AC 2009-1575: ELECTRIC GENERATOR FOR WIND OR HUMAN POWER
}

\section{Horacio Vasquez, University of Texas, Pan American}

Dr. Horacio Vasquez is an Assistant Professor in the Mechanical Engineering Department at the University of Texas-Pan American (UTPA), in Edinburg, Texas. His current research interests are in the areas of control systems, mechatronics, renewable energy, and engineering education.

\section{Carlos Gomez, University of Texas, Pan American}

Carlos Gomez is an undergradaute mechanical engineering student at the University of Texas-Pan American. He enjoys working with Mechatronics systems and is involved with the minibaja SAE team at UTPA. 


\title{
Construction and Testing of an Electric Generator for Wind or Human Power
}

\begin{abstract}
One of the most important engineering challenges nowadays is the developing and implementation of renewable energy systems that are practical, cost effective, and bring benefits to the society as well as to the environment. In general, wind generators operate satisfactorily at certain wind speeds which are rarely present in some geographic locations, and at other than the rated speed, they have poor performance. A permanent magnet three-phase alternating current (AC) generator was built in this project and it allows modifying its design and experimenting with several operating and control strategies at different speeds and load configurations. The performance of the generator was determined while charging deep-cycle batteries, and promising results were obtained. The generator is intended to be powered by wind or human power. For the human power application, a cyclist generates electricity by riding a regular mountain bicycle whose transmission was connected to the shaft of the generator. The bike becomes a stationary exercise machine because the rear wheel is lifted so that it does not have contact with the ground. Therefore, part of the energy burned during bicycling is stored in batteries instead of wasting it as it commonly happens with stationary bikes. The output of the three-phase AC generator was connected to a rectifier to obtain direct current (DC) voltage to charge the batteries. The main purpose of this paper is to present the experimental results obtained with this generator constructed as part of an undergraduate research project by a Mechanical Engineering student. Future work includes characterizing other configurations of the stator and designing a control system algorithm to optimize energy conversion not only to charge deep-cycle batteries but also to power loads connected to the batteries in a simultaneous or alternating way. Additional results are going to be obtained through experimental research to design, model, and optimize this relatively low-power electric generator propelled by wind or human power. The generator is developed for these specific applications taking into account the power limitations of such energy sources but considering as a very important task to harvest this energy for long time periods to benefit our society as well as the environment.
\end{abstract}

\section{Introduction}

Wind generators could be good electricity providers when moved by other means as long as there is enough power to rotate them in the neighborhood of their rated speed and to sustain the torque required to continuously operate them under those conditions for a long time. Human power could be used to propel these generators; but, the maximum power a person is able to generate for several minutes while exercising is in the order of 80-120 W at the optimal rate of about $60 \mathrm{rpm}$, which depends on the athletic ability and training of the individual ${ }^{1}$. Power losses in the mechanical transmission between the crankset of the bike and the generator need to be minimized by avoiding rubbing parts and using some sort of lubrication on moving parts. A few people taking turns to pedal a bike could store useful energy to power lights and preferably other energy efficient devices. In most well-off electrified cities and urban communities, because of needed exercise activities for individuals, there are homes, hotels, schools, and gyms with bikes 
and other exercise machines that are propelled long time periods everyday; therefore, there is an opportunity to harvest the human energy that is being burned anyways. In contrast, in many rural, isolated and/or poor communities worldwide, there is not enough electricity or not electricity at all; hence, there are opportunities to install systems in such places to harvest energy from multiple sources such as wind, solar, and human power. Consequently, there are opportunities everywhere for alternative energy sources to improve the quality of life.

Using a generator rated at much higher power than the one a person can continuously produce is not adequate in human power applications. When the operating velocities of the generator are higher than the optimal pedaling rate of an individual, a transmission system with adequate gear ratios is needed to achieve the desired generator speed. Therefore, power required to propel the generator has to be available in an acceptable combination of speed and torque. An engineering approach involving system design and experiments is required to obtain satisfactory results when integrating a source of power, generator, electric loads, and/or energy storage devices. Using individual components and connecting them together, without carefully doing analysis and experimenting with some options, would rarely create a system that works in a satisfactory way. While searching for commercially available low-power (100 W or so) wind generators that could be used for human power and low-speed wind applications, it was determined that some of them are self-regulated and limited to a battery voltage configuration (e.g. $12 \mathrm{~V}$ or $24 \mathrm{~V}$ ), which make them not practical for this project. Other generators are made to operate at rated wind speeds that are almost never present in some geographic locations, and at other than its rated wind speed, they have poor performance. As an alternative, a generator for low-speed wind or human power applications that allows varying its design and control parameters in order to obtain optimal performance was constructed and tested in this project. The basic configuration of the generator was adopted from a small wind generator book by Piggott $^{8}$; but, it was slightly modified, constructed, and tested. Its performance when charging deep-cycle batteries was determined in order to identify optimal operating conditions that maximize output power at different speeds. At first, a stationary mountain bicycle was connected to the generator; nevertheless, for convenience to perform multiple experiments in the lab, an AC motor was connected to drive the generator at different constant speeds. The generator is going to remain connected to the bicycle and used by students for demonstrations and to study concepts in electromechanical, instrumentation, and renewable energy systems.

It was found in the literature that several undertakings have also made efforts to develop lowspeed wind generators or to develop other human powered devices that generate electricity. For example, Reed et al. ${ }^{2}$ modeled, simulated, constructed, and tested a similar generator with 6 stator phases and 24 magnets per rotor disk; they obtained about $240 \mathrm{~W}$ when rotating the generator at approximately $350 \mathrm{rpm}$. Pandian ${ }^{3}$ proposed a new method to generate electricity at children playgrounds taking advantage of large number of children that, unaware of their free work, happily would propel the mechanisms that generate electricity. Pandian ${ }^{3}$ built a prototype see-saw with pneumatic cylinders that compress air and is used to propel a DC motor working as a generator; the total efficiency of the system was estimated at $1.6 \%$ from human power input to electricity output; which the author indicated that is comparable to an energy generating piezoelectric shoe device. To emphasize the importance of these renewable energy projects, Zahnd et al. ${ }^{4}$ explained how in isolated villages, communities, and colonies in Nepal and many other countries, about 2 billion people endure harsh living conditions in part because of lack of 
electricity. For instance, those people prepare meals on open fireplaces that require burning logs inside their houses, generating smoke clouds that affect their health. Deforestation problems are also caused not only because of the logs needed for the fireplaces but also due to tree resin extraction needed to create candles for lighting. As an example of electricity generation in a small scale, Zahnd et al. ${ }^{4}$ installed a $150 \mathrm{~W}$ hydropower generator to provide 30 houses with three 1W white LED lights, which seems to be a small deed but it produces a significant benefit for those people. Electricity is difficult and expensive to store and this is one of the main limitations nowadays to implement reliable renewable energy systems that use solar and wind power sources $^{5}$. Even though lithium-ion batteries have better performance than lead batteries, the relative low cost of lead batteries make them better suited for high-energy storage applications ${ }^{5}$. As another example, Valdes et al. ${ }^{6}$ developed a low technicality vertical shaft wind generator made with a Savonius-type rotor that looks like a split-open cylinder forming the rotor blades. Such generator is supported by a bamboo frame, its shaft is made from concrete, and the bearings are made with glass marbles rolling on carved hard wood mountings. A bicycle wheel is connected to the lower end of the shaft and it is in contact to propel numerous bicycle dynamos connected to charge a $12 \mathrm{~V}$ car battery ${ }^{6}$. Even though this low technicality generator is a great asset in isolated poor communities around the world (Madagascar, in particular) and it only costs about $\$ 100$, it requires high maintenance of the dynamos making the price of a $\mathrm{kW} \cdot \mathrm{h}$ high; but, given their situation, there is not other electrification option ${ }^{6}$. Corbyn ${ }^{7}$ also reported the developing and installation of $500 \mathrm{~W}$ and $1 \mathrm{~kW}$ small wind generators developed based on the book of $\operatorname{Piggott}^{8}$ as alternative or complement to costly photovoltaic arrays needed in water pumping application in isolated communities in Philippines. First, they store the energy in a battery bank and then use an DC-to-AC inverter to power a submersible pump inside a well to fill an elevated tank that distributes water to neighborhoods. Other efforts using most sophisticated equipment and advanced control techniques are also being developed and tested by other scientists and engineers. For instance, Ogawa et al. ${ }^{9}$ used a switched reluctance generator for a small size wind power generating system for which two complementary control strategies were required, one to maintain constant resistance load and another to obtain maximum power point tracking (MPPT) operating conditions. Also, Amano ${ }^{10}$ developed a MPPT for a small wind generator based on adjusting the velocity of the rotor to a predetermined velocity that is a function of the wind speed. Amano's controller consists of a PID that adjusts a $20 \mathrm{kHz}$ PWM signal firing a transistor to regulate the current flowing from the generator to the battery to allow regulating the angular velocity of the generator ${ }^{10}$.

This project performs experimental research to design, model, and optimize an electric generator propelled by alternative energy sources, like wind or human power, and this paper presents the results obtained with the first prototype that has been constructed and tested. The electric generator was developed considering a significant accomplishment to harvest energy for long time periods to benefit our society as well as the environment. This is considered an important step in an ongoing project that requires multiple additional steps to be satisfactorily completed. The performance of the prototype three-phase AC generator was determined under multiple operating conditions. The shaft of the generator was attached to a three-phase AC motor with a variable frequency drive to control the velocity of the generator and study its performance while charging batteries in different voltage configurations. This setup is also useful to make demonstrations and study concepts in power transmission, renewable energy generation, storage, and utilization. I summary, the generator has two main purposes: a) to be connected to the 
transmission of a bicycle so that a cyclist can generate electricity, just by riding a regular bike that becomes a stationary exercise machine, and b) connect it to a propeller to harvest energy at low-wind speeds. Hence, this project intends to impact two modern society concerns in an integrated way: usage of alternative energy sources and developing of attractive human exercise activities. For isolated villages and colonies around the world, and even in developed countries, this project presents some ideas to supply them with electricity.

\section{Design and Construction of the Generator}

A permanent magnet three-phase alternating current (AC) generator was constructed to experiment generating electricity with wind or human power. The configuration chosen for the generator consisted of three stator phases, each of which made up by two coils, and eight magnets mounted on each of two rotor disks fixed to the same shaft that rotate so that the magnetic fields created by the magnets cross the stator coils to induce electric fields in the coils. This generator is one of the configurations presented by Piggott ${ }^{8}$ which was slightly modified due to construction difficulties and variations. Piggott's book presents the procedure to construct the generator. The magnets are located in an alternating south-north pole array around the rotor disks; the magnets on one disk face the magnets on the other disk in such a way that they attract each other. Figure 1 presents a computer drawing of the generator constructed in this project. The software used to make this drawing allows rotating the disks in order to determine the location of the magnets with respect to the coils on the stator at any angular position.

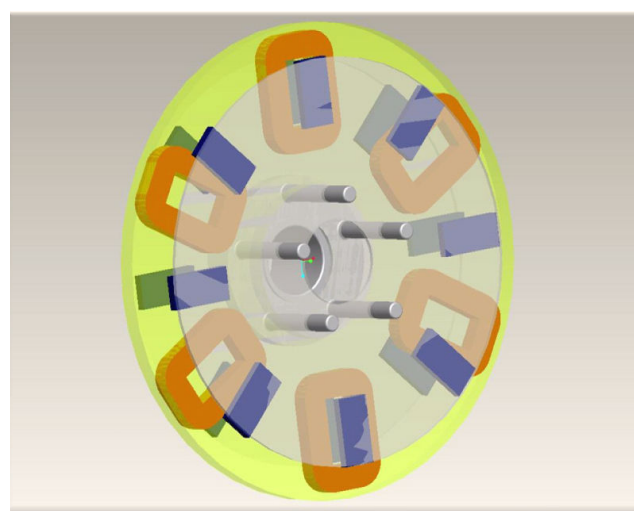

Figure 1. Drawing of the generator. 
The stator coils were connected to create a three-phase configuration and the output of the phases are connected to a bridge rectifier, as shown in Figure 2, to obtain direct current (DC) voltage to charge deep-cycle batteries.

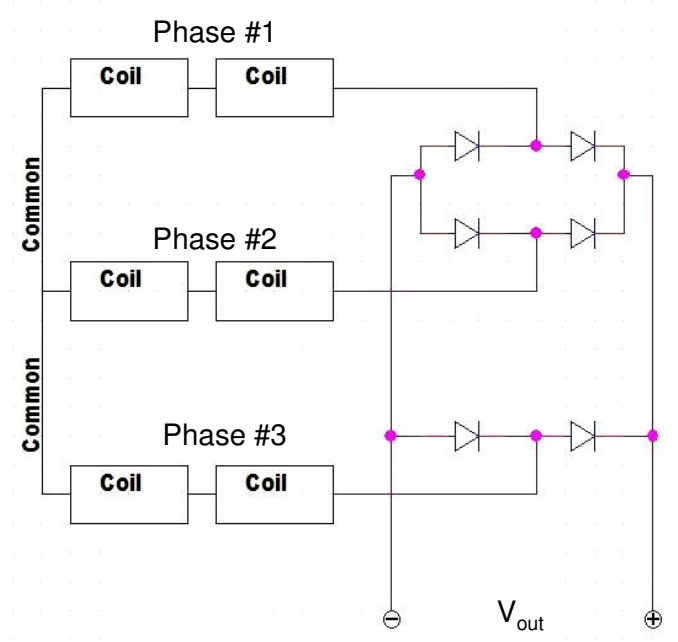

Figure 2. Connections of the stator coils and bridge rectifier.

Figure 3 shows the generator with 8 pairs of Neodymium Iron Boron $(\mathrm{NdFeB})$ magnet blocks, which were assembled facing each other on two rotor disks located at both sides of the stator, and placed in an alternating south-north pole array around the disks. The magnets are attached to steel disks, which rotate forcing the magnetic field created by the magnets to cross copper coils in the stator, fixed between the disks, and as a consequence electricity is generated.

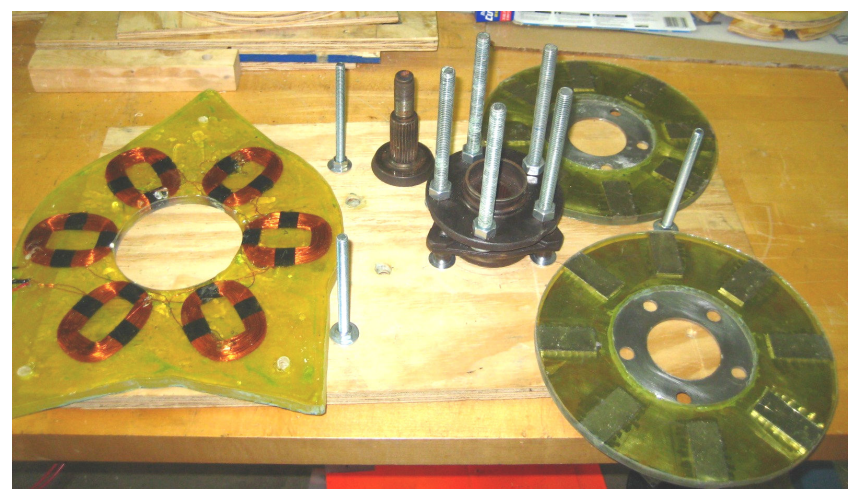

Figure 3. Components of the constructed AC generator.

The main components of the generator consist of the rotor and stator. The rotor is made with two sets of eight magnets mounted on $1 / 8^{\prime \prime}$ thick, 10 inch diameter, steel disks. Piggott ${ }^{8}$ recommends $1 / 4$ inch thick disks, $1 / 2$ inch thick magnets, and 9 inch diameter rotor disks, but difficulties were found to assemble the generator with those dimensions and some modifications 
were required. The stator consists of six coils of 120 loops of magnet wire connected in a three phase configuration with a pair of coils forming each phase. The stator was molded in fiberglass to form a rigid structure, prevent misalignment, and to protect the coils from humidity and corrosion. The disks were mounted on a car bearing hub and the stator was placed between the rotors to create an axial magnetic flux machine.

\section{Experimental Setup}

The generator was initially connected to the transmission of a regular mountain bike as shown in Figure 4. The mountain bike has 21 gear ratios but because an additional chain was connected around the largest cog in the cassette of the bike to a cog attached to the shaft of the generator, 6 of those gear ratios are no longer available. The original shifters and derailleurs of the bike are used to change to any of the available 15 gear ratios which provide a good combination of speeds for this application. Note in Figure 4 that if, for example, the gear ratio $\mathrm{r}_{1^{-}}$ to- $r_{2}$ between the crank and the cassette is 4-to- 1 and also the gear ratio $r_{3}$-to- $r_{4}$ is 2-to-1, the cyclist must pedal at $50 \mathrm{rpm}$ in order to rotate the generator at $400 \mathrm{rpm}$. This is assuming that the cyclist can sustain the power required to move the generator at such speed, which is going to depend to a great extent on its design and performance characteristics.

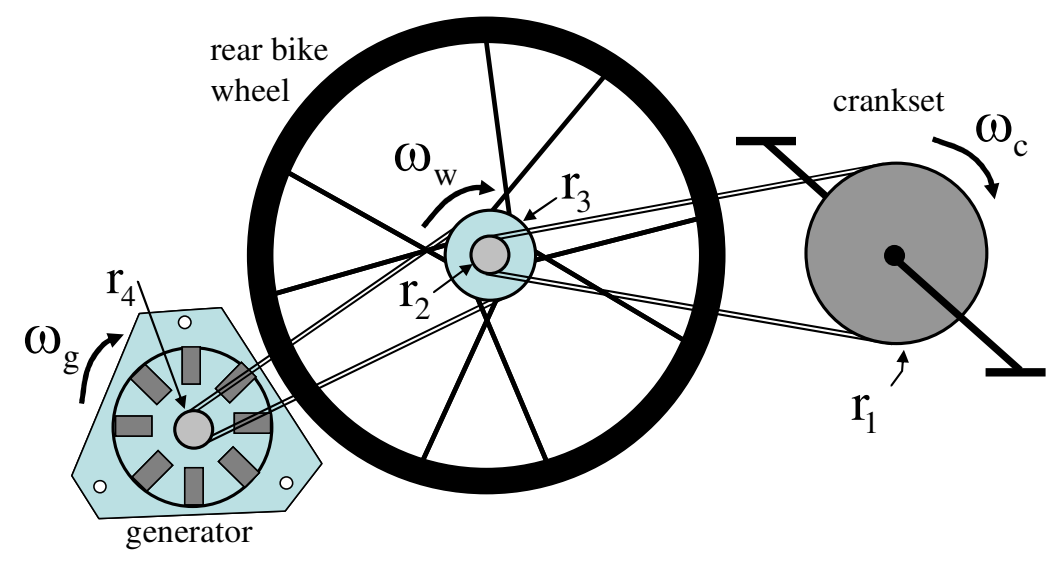

Figure 4. Bike transmission and connection to generator.

After removing the generator from the bike transmission, another experimental setup consisting of the generator, belt and pulleys, three-phase induction motor, variable frequency drive, and deep-cycle batteries was created. As shown in Figure 5, for convenience of studying the performance of the generator at different angular speeds, it was driven using an induction motor whose velocity can be easily adjusted. 

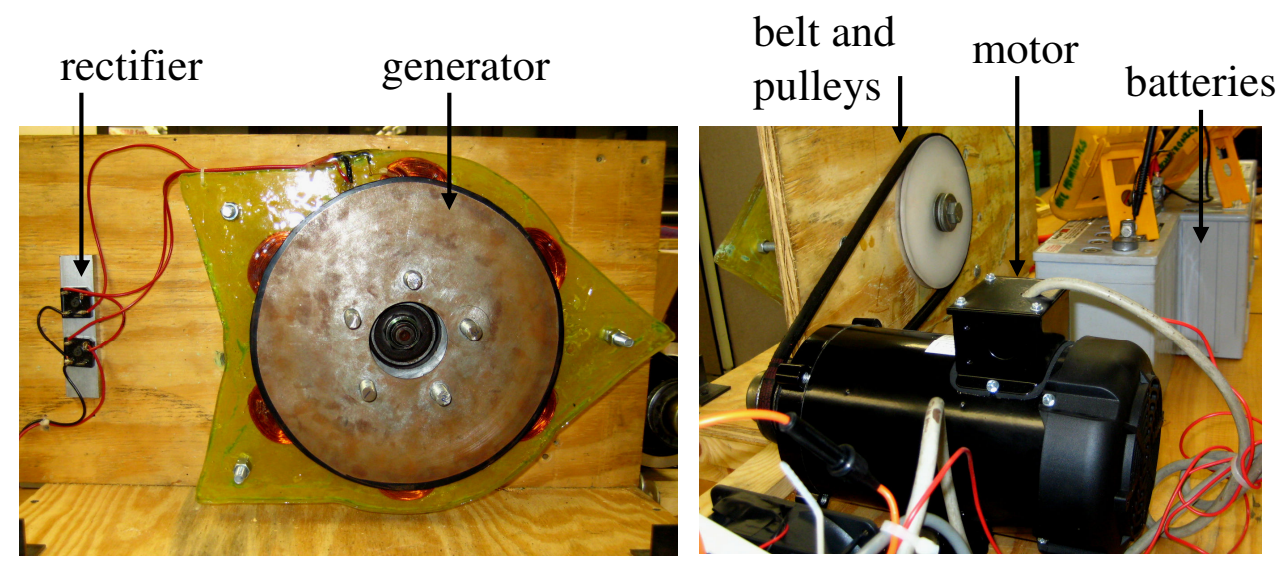

Figure 5. Experimental setup

\section{Experimental Results}

At first, the generator was coupled to the transmission of a mountain bike as presented in Figure 4 and connected to $24 \mathrm{~V}$ deep-cycle batteries (two $12 \mathrm{~V}$ batteries in series). It was determined that a person could pedal the bike for long time periods generating power up to approximately $120 \mathrm{~W}$ depending on pedaling effort and gear ratio being used. Generating 25-30 $\mathrm{W}$ is relatively easy and it is achieved even by turning the pedals with the hand. Also, pedaling using the highest gear ratio of about 8-to-1 is relatively difficult at generator speeds higher than $450 \mathrm{rpm}$. It was also determined that friction due to rubbing parts between the generator shaft, bearing case, and the supporting structure of the generator creates considerable power losses that need to be avoided to have a smooth rotation of the generator and transmission system. This can easily be determined by rotating the system when the generator output is not connected to anything, and rotation should be easy and smooth.

The open-loop voltage was measured at the output of the bridge rectifier at different angular velocities of the generator. Figure 6 shows the open-loop voltage versus angular velocity; note that the voltage is directly proportional to the angular velocity and it is about $70 \mathrm{~V}$ at $400 \mathrm{rpm}$.

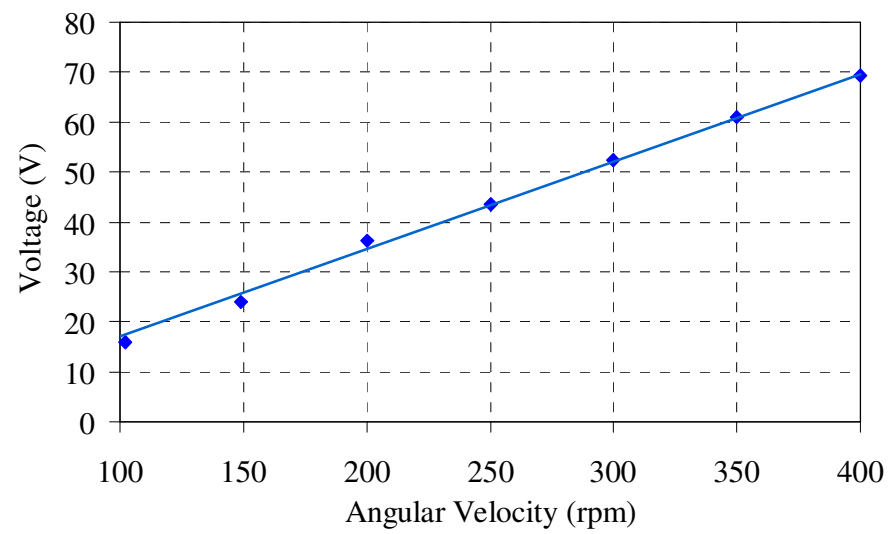

Figure 6. Open-loop voltage versus angular velocity. 
In order to determine the most adequate battery charging operating conditions of the generator, a single $12 \mathrm{~V}$ deep-cycle battery was connected to the output of the bridge rectifier. It was determined that the battery starts charging at about $60 \mathrm{rpm}$, and a current of about 4 A flow into the battery at $400 \mathrm{rpm}$. These results are presented in Figure 7. Similar experiments were performed using 2, 3, and 4 deep-cycle $12 \mathrm{~V}$ batteries in series. Even though charging higher voltage battery configurations starts occurring at higher generator velocities, at the same speed, the current flowing into the batteries is lower at higher battery voltage configurations; this is convenient to prevent failure when thin magnet wire is used to make the stator coils.

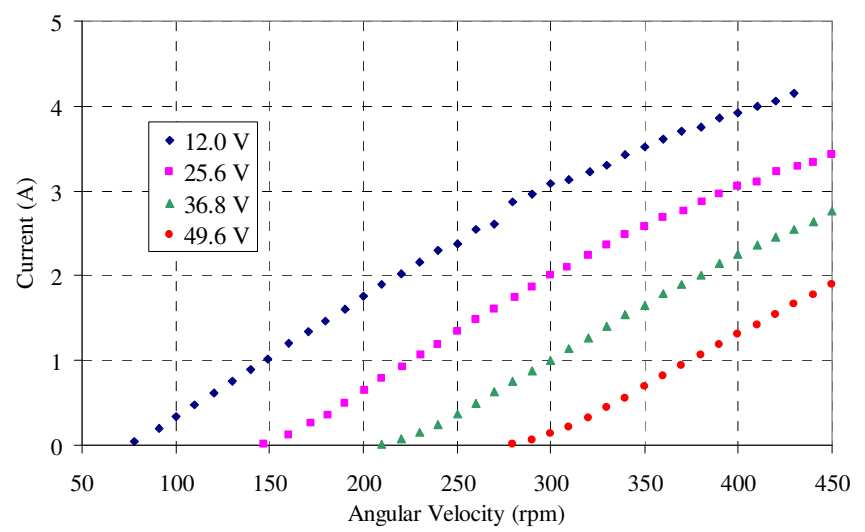

Figure 7. Current output versus velocity of generator while charging deep-cycle batteries

Interesting results were obtained plotting the power going into the batteries versus the angular velocity of the generator at different battery voltages. It was determined that at any generator speed, there is a battery configuration that maximizes the power being transferred into the batteries. Figure 8 presents the most important results obtained in this project, indicating that the best battery charging voltage at velocities less than $220 \mathrm{rpm}$ is $12 \mathrm{~V}$; between 220 and 380 $\mathrm{rpm}$ is $24 \mathrm{~V}$, and between 380 and $450 \mathrm{rpm}$ is $36 \mathrm{~V}$; at higher velocities, higher voltages are more convenient.

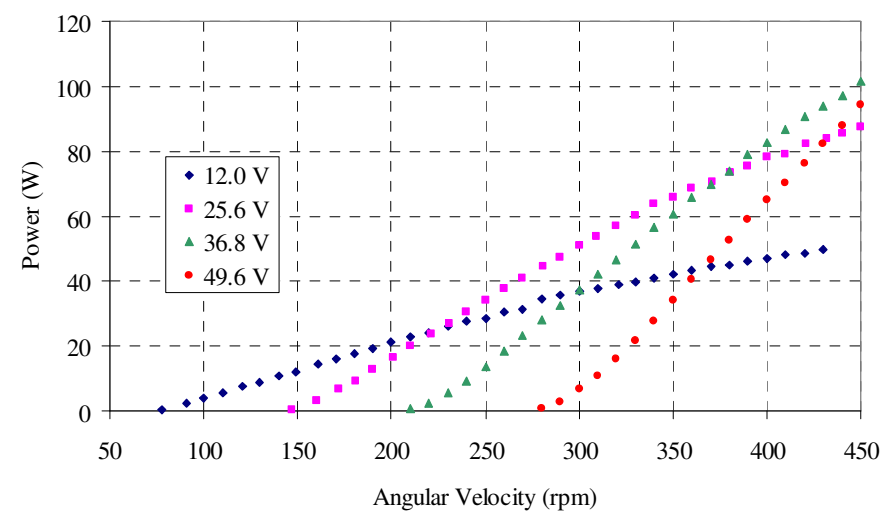

Figure 8. Power output versus velocity of generator while charging deep-cycle batteries 
Of particular interest is the operating point of the generator at $400 \mathrm{rpm}$ that allows charging $24 \mathrm{~V}$ or $36 \mathrm{~V}$ batteries at about $80 \mathrm{~W}$. It was proven that an individual pedaling a bicycle is able to propel the generator with medium effort (depends on the person) at this operating point and 80 $\mathrm{W}$ are transferred into the batteries. More or less power can be transferred into the batteries but that depends on the individual pedaling and the gear ratio being used. However, at the same 400 rpm of the generator, if the battery configuration voltage is $48 \mathrm{~V}$, the power transferred into the batteries is about $65 \mathrm{~W}$; and if the voltage is $12 \mathrm{~V}$, the power is about $46 \mathrm{~W}$. The open-loop voltage at $400 \mathrm{rpm}$ is about $70 \mathrm{~V}$. These results demonstrate that there are charging battery voltage configurations that are more suitable than other to transfer energy to the batteries from a generator at different speeds.

\section{Future Work}

Pilot results have been obtained to justify the potential of this project; additional theoretical and exploratory work is required to confirm that a practical electricity generating station might be developed for human power and similar applications. The performance of the generator will be monitored with a data acquisition system to display the operating conditions on a computer screen so that the cyclist can see the average power being generated, angular velocity, state of charge (SOC) of the battery, and other operating conditions. In addition, a propeller is going to be designed and constructed to be installed on a similar generator for low-speed wind applications.

Future work will also include modeling the generator's performance using nonlinear techniques, such as artificial neural networks (ANN) to correlate design and input parameters with output power. The importance of the model is that it could be used for simulations to estimate the output power of the generator under multiple design and input conditions. An ANN is a conglomerate of computational units, or neurons, organized in layers. An important characteristic of an ANN is that all the neurons can be trained, under supervised or unsupervised learning, in an inter-dependent way, to associate, learn, and/or classify information. Most of the difficulty experienced to fit nonlinear data is due to inherent variability as well as non-linear nature of the inputs and outputs involved in a process. The literature is clear in pointing out that ANNs can adequately be trained to learn and estimate non-linear data originated from measurements ${ }^{11}$. However, for the trained ANN to be useful, a high degree of repeatability of the system that generated the original data is required. In order to train ANNs, back propagation techniques could be used ${ }^{12}$. Furthermore, using sophisticated software packages, like Matlab, make it possible to train neural network in practical ways.

\section{Conclusions}

A three-phase generator was constructed and tested for wind or human power applications, and promising results have been obtained with open-loop output voltages above $18 \mathrm{~V}$ for angular velocities higher than $115 \mathrm{rpm}$. Also, $80 \mathrm{~W}$ were transferred into $24 \mathrm{~V}$ (also into $36 \mathrm{~V}$ ) batteries at $400 \mathrm{rpm}$. This is a velocity and power that a human can reasonable create while exercising. It was determined that at any generator velocity, there is a range of battery voltage configurations 
that maximizes the power being transferred into the batteries. The generator is going to remain connected to the bicycle for instruction and demonstration purposes in the areas of electricity generation, power transmission, and energy storage. With the developed system, an individual can easily feel the effort required to generate and transfer energy into deep-cycle batteries. Therefore, the first prototype of a stationary exercise machine have been developed to allow part of the energy burned by a cyclist to be stored in batteries instead of wasting all of it as it commonly happens. Many applications could be found for the generator developed in this project. Future work is required to study other generator configurations and to model and

simulate it. Future work will also be performed to construct an adequate propeller to test the generator under low-wind speed conditions.

\section{Acknowledgements}

The authors would like to express appreciation to the Office of the Interim Vice Provost for Graduate Studies, Undergraduate Research Initiative program at UTPA for the financial support to develop this undergraduate research project.

\section{References}

[1] Cho, Chang K.; Yun, Myung Hwan; Yoon, Chang S.; Lee, Myun W. 1999. Ergonomic study on the optimal gear ratio for a multi-speed bicycle. International Journal of Industrial Ergonomics, v 23, n 1-2, January, p 95-100

[2] Reed, Justin; Venkataramanan, Giri; Rose, Jonathan. 2008. Modeling of battery charging wind turbines. 7th International Conference on Power Electronics, p 1121-1126

[3] Pandian, Shunmugham R. 2004. A human power conversion system based on children's play. 2004 International Symposium on Technology and Society, p 54-61

[4] Zahnd, Alex; Kimber, Haddix McKay. 2009. Benefits from a renewable energy village electrification system. Renewable Energy, v 34, n 2, February, 2009, p 362-368.

[5] Ibrahim, Hussein; Ilinca, Adrian; Perron, Jean. 2007. Comparison and analysis of different energy storage techniques based on their performance index. IEEE Canada Electrical Power Conference, p 393-39

[6] Valdes, L.-C.; Darque, J. 2003. Design of wind-driven generator made up of dynamos assembling. Renewable Energy, v 28, n 3, March, p 345-362

[7] Corbyn, Andrew. 2007. Small wind-turbine community-based renewable energy systems in the Philippines. Wind Engineering, v 31, n 5, p 353-361

[8] Piggott, Hugh. How to build a wind turbine? The axial flux windmill plans. 2005. 
[9] Ogawa, K.; Yamamura, N.; Ishda, M. 2006. Study for small size wind power generating system using switched reluctance generator. Proceedings of the IEEE International Conference on Industrial Technology, p 1510-1515

[10] Amano, Yoko; Kajiwara, Hiroyuki. 2006. Maximum output power control for variablespeed small wind turbine generator system. 2006 SICE-ICASE International Joint Conference, p 6069-6074

[11] Poopalasingam, S., Reeves, C.R., and Steele, N.C. 1994. Application of neural networks for sensor performance improvement. Neural Networks for Signal Processing IV, Proceedings of the 1994 IEEE, 633-640.

[12] Hagan, M., Demuth, H., and Beale, M. 1995. Neural Network Design. Boston: PWS Publishing. 